\title{
AGN Singularities and Jets Modelled with the Superstar Scenario
}

\author{
Ding-Yu Chung \\ Utica, Michigan, USA \\ Email: dy_chung@yahoo.com
}

How to cite this paper: Chung, D.-Y. (2019) AGN Singularities and Jets Modelled with the Superstar Scenario. International Journal of Astronomy and Astrophysics, 9, 142-153. https://doi.org/10.4236/ijaa.2019.92011

Received: April 1, 2019

Accepted: June 18, 2019

Published: June 21, 2019

Copyright $\odot 2019$ by author(s) and Scientific Research Publishing Inc. This work is licensed under the Creative Commons Attribution International License (CC BY 4.0).

http://creativecommons.org/licenses/by/4.0/ (c) (i) Open Access

\begin{abstract}
In this paper we present solutions with the superstar scenario for the problems of singularity and the relativistic jet in AGN (Active Galactic Nuclei) based on supermassive black hole with singularity. The five-zone structure of superstar from inside to outside consists of the Singularity-Free Superstar Core (SC), the short-range repulsive super force field (SFF) near the event horizon, the superstar lepton sphere (SLS) containing infalling leptons (electron-positron pairs), the superstar ergosphere (SE), and the superstar accretion disk (SAD). As in the Meissner effect in superconductor, the short-range SFF repulses leptons in the SLS preventing singularity, while infalling leptons from the SAD and the SE continue to enter the SLS through the strong gravity of the SC. When the density at the bottom of the SLS reaches the critical density, leptons fall into the SC with the corresponding size increase of the SC to prevent singularity. Without further infalling leptons, the short-range repulsive force from the SFF disintegrates the SLS into the SLS plasma fragments (electron-positron pair plasma), detaching from the SC. Some SLS plasma fragments in the SAD generate the broad relativistic SAD jet, and some SLS plasma fragments in the SE generate the coincident narrow relativistic SE jet. In this two-jet model (the origin of the spine-sheath jet structure), protected by the SAD jet, the fast and narrow SE jet inside the slow and broad SAD jet generates the VHE (very high energy $\geq 100 \mathrm{GeV}$ ) Synchrotron Self Compton (SSC) gamma-ray emission without the attenuation by the photons in the BLR (broad line region) of flat spectrum radio quasar (FSRQ). In conclusion, AGN based on supermassive superstar provide the solutions for singularity, VHE gamma-ray emissions in FSRQs and FR1 type radio galaxies, AGN jet structure, and AGN jet type.
\end{abstract}

\section{Keywords}

ANG, Relativistic Jet, Superstar, Supermassive, Singularity, Black Hole, Super Force Field, Very High Energy Gamma-Ray Emission 


\section{Introduction}

AGN (Active Galactic Nuclei) is based on supermassive supermassive black hole with singularity. Black hole has been a standard model for the collapse of a large star. Singularity in black hole remains contentious. Gravastar (gravitational vacuum star) [1] [2] by P. O. Mazurand and E. Mottola is a model for the collapse of a large star without singularity. In gravastar, quantum effects would change space-time around a collapsing star, initiating a radical phase transition like when liquid water becomes ice, for some of the infalling matter. For gravastar, the phase transition involves the transformation into a "gravitational vacuum" with an interior de Sitter condensate surrounded by a Bose-Einstein condensate (BEC) bubble, similar to the transformation of a cloud of atoms into one huge "super-atom", a BEC at an extremely low temperature above absolute zero degree. The BEC is prevented from complete collapse by the interior de Sitter condensate exerting a balanced pressure outwards on the condensate. A thin phase boundary (shell) for the phase transition is in between the interior region and the exterior region.

In this paper, superstar without singularity is proposed as an alternative to black hole and gravastar as described in the previous paper [3]. Due to quantum effects, the space-time around gravastar exerts a balanced pressure outwards on gravastar to prevent the complete collapse of gravastar. Similarly, due to quantum effects, the space-time around superstar is the short-range repulsive super force to prevent the complete collapse of superstar. The repulsive effect of the super force field at nearly infinite density is like the repulsive Meissner effect in superconductor to eject applied magnetic fields from the interior of the superconductor at nearly absolute zero temperature as described in the previous paper [4].

This paper describes AGN based on supermassive singularity-free superstar as an alternative to AGN based on supermassive black hole with singularity. AGN produce very high luminosities in a very concentrated volume. In the prevailing model of the physical structure of AGN, at the center is a supermassive black hole $\left(10^{6}\right.$ to $\left.10^{10} \mathrm{M}_{\ltimes}\right)$ whose gravitational potential energy is the ultimate source of the AGN luminosity [5]. Matter pulled toward the rotating supermassive black hole form a rotating accretion disk. The region near the accretion disk is the broad line region (BLR) with high-density optical and ultraviolet photons emitted from the accretion disk. The region much farther from the disk is the narrow line region with low-density photons. Outflows of energetic particles in the relativistic collimated jet occur along the poles of the disk. A subset of the jetted AGN is blazar [6] with the relativistic jet closely aligned to the line of sight of the observer. Blazars show the properties of variability at all frequencies-time scales and high polarization at both optical and radio frequencies. Blazars include flat spectrum radio quasars (FSRQs) that show broad optical emission lines and BL Lacertae objects (BL Lacs) with weak or even absence of such emission lines.

The spectral energy distribution (SED) of blazars is dominated by the gam- 
ma-ray emission, but the location of the main gamma-ray emitting region in blazars is still uncertain. In the standard scenario of model, the SED of the large majority of gamma-ray detected FSRQ is the BLR External Compton (EC) scenario [7] [8] where the BLR provides effective seed photons for the inverse Compton (IC) scattering by relativistic electrons in the jet. In the recent paper by Costamante, Cutini, Tosti, Antolini, and Tramacere, the BLR-EC scenario is tested [9]. They test this scenario on the Fermi gamma-ray spectra of 106 broad-line blazars detected with the highest significance or largest BLR, by looking for cut-off signatures at high energies compatible with $\gamma-\gamma$ interactions with BLR photons, resulting in the attenuation of gamma-ray. They found that only one object out of ten seems compatible with substantial attenuation through the interaction with BLR photons. The one object is below the cut-off energy. They conclude that for 9 out of 10 objects, the jet does not interact with BLR photons to produce gamma-ray. The result is the production of very high energy (VHE, $\geq 100 \mathrm{GeV}$ ) gamma-rays without attenuation, contradicting the BLR-EC scenario. Such VHE gamma-rays have to be produced outside the BLR. However, it is difficult to find a scenario without the involvement of the BLR in FSRQ where the BLR is highly active. As a result, the proper relativistic jet producing VHE gamma-ray emission in FSRQ is still unknown.

This paper proposes that AGN based on supermassive singularity-free superstar provide the solutions for the problems of singularity and the relativistic jet to producing VHE gamma-ray emission in AGN based on supermassive black hole. Section 2 describes black hole and superstar. Section 3 deals with AGN based on supermassive superstar,

\section{Black Hole and Superstar}

The uncertainty principle in quantum mechanics

$$
\sigma_{x} \sigma_{p} \geq \frac{\hbar}{2}
$$

Postulates that the position, $X$, and momentum, $p$, of a particle cannot be simultaneously determined with arbitrarily high precision. In the generalized uncertainty principle [10], an extra term related to the Planck energy is introduced to avoid singularity.

$$
\sigma_{x} \sigma_{p} \geq \frac{\hbar}{2}+\frac{\alpha G}{c^{3}}
$$

where $\alpha$ is a constant and $G$ relates to the Planck energy, At energy much below the Planck energy, the extra term in Equation (2) is irrelevant and the Heisenberg uncertainty principle is recovered, while approaching the Planck energy, the extra term becomes relevant, and is related to the minimal observable length. In this paper, an extra force field is introduced to maintain the non-zero minimal length and momentum at the extreme conditions such as at the temperature near absolute zero degree $\left(\sigma_{p} \approx 0\right)$ and extremely high density $\left(\sigma_{x} \approx 0\right)$ when $\sigma_{x} \sigma_{p}$ $\approx 0$. This extra force field is the short-range super force field, resulting in su- 
perstar and superconductor [3] [4]. Superstar with the short-range super force field to star is like superconductor with the short-range super force field to conductor [4].

The conventional five-zone structure from inside to outside of black hole [11] consists of singularity at the center of black hole,, the event horizon [12], the black hole photon sphere (BHPS) [13], the black hole ergosphere (BHE) [14], and the black hole accretion disk (BHAD) [15] as in Figure 1.

Singularity is the region of the black hole where all the mass of the black hole has been compressed down to nearly zero volume with almost infinite density and an enormous gravitational force. As the boundary of the black hole, the event horizon with the Schwarzschild radius preventing any escape of matters and radiation. Adjacent to the event horizon, the surrounding unstable and thin black hole photon sphere (BHPS) with a radius 1.5 times the Schwarzschild radius contains photons travelling in a circular orbit. Outside of the event horizon, a rotating black hole produces black hole ergosphere (BHE) where nothing can remain at rest. Ergosphere has an elliptical shape like a flattened sphere, and the north and south poles of the ergosphere touch the top and bottom of the event horizon. Objects and radiation can escape from ergosphere.

Black hole accretion disk (BHAD) is mostly gas around black holes transports gas to the black hole at their centers. The gas is pulled into the BHAD from infalling star and interstellar material, and the latent angular momentum of gas causes the BHAD to rotate in the shape of pancake. The accreting gas near the black hole event horizon orbits at very high speeds to produce friction, which heats it up so much that individual atoms dissociate into plasma consisting of mostly leptons (electron-positron pairs), some of which fall into black hole. The plasma creates a strong magnetic field around black hole. The BHAD is larger than the BHE, so there is space between the BHAD and the BHE. The region near the accretion disk is the broad line region (BLR) with fast-moving high-density optical and ultraviolet photons emitted from the accretion disk. The region much farther from the disk is the narrow line region with slow-moving low-density photons.

The five-zone structure of superstar is similar to the five-zone structure of black hole. The five-zone structure of superstar from inside to outside consists of the Singularity-Free Superstar Core (SC), the super force field (SFF) as the short-range repulsive force field near the event horizon to separate the SC and

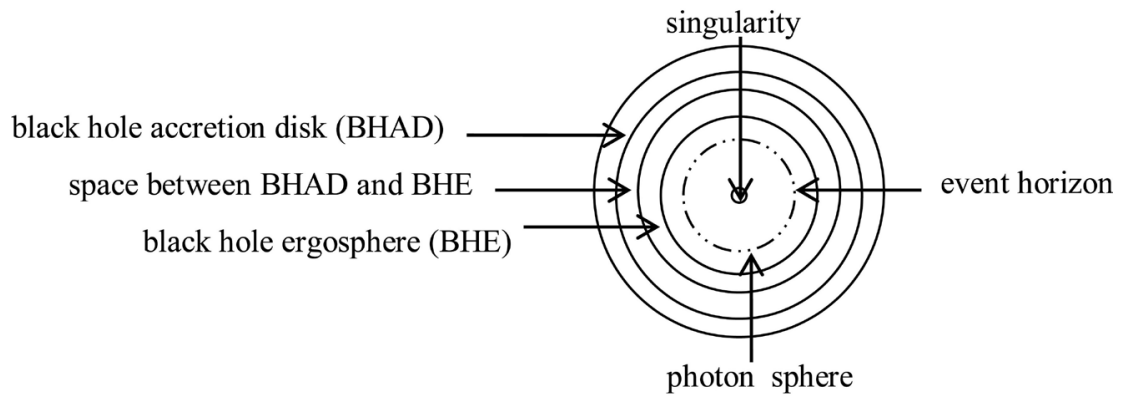

Figure 1. The structure of black hole. 
the infalling leptons, the superstar lepton sphere (SLS) to contain infalling leptons, the superstar ergosphere (SE), and superstar accretion disk (SAD) as in Figure 2.

The Schwarzschild radius of a rotating black hole is given as

$$
r_{s}=\frac{G M}{c^{2}}
$$

where $G$ is the gravitational constant, $M$ is the object mass, and $c$ is the speed of light. The radius of the superstar core is equal to or larger than the Schwarzschild radius.

$$
r_{\text {superstar core }} \geq \frac{G M}{c^{2}}
$$

The Sun as a superstar has the mass of $1.99 \times 10^{30} \mathrm{~kg}$ and the Schwarzschild radius of $2.95 \times 10^{3} \mathrm{~m}$. Supermassive superstars up to 21 billion $\left(2.1 \times 10^{10}\right)$ of the mass of the Sun have been detected, such as NGC 4889 with the mass of 4.2 $\times 10^{40} \mathrm{~kg}$ and the Schwarzschild radius of $6.2 \times 10^{13} \mathrm{~m}$.

\section{AGN Based on Supermassive Superstar}

The Meissner effect in superconductor is explained by the outward pressure of the super force field to eject applied magnetic fields from the interior of the superconductor as it transitions into the super force field at nearly absolute zero temperature. As in the Meissner effect in superconductor, the super force field in supermassive superstar exerts a short-range repulsive force to repel leptons in the SLS, while infalling leptons from the SAD and the SE continue to enter the SLS through the strong gravity of the SC. When the density at the bottom of the SLS reaches the critical density, leptons fall into the SC with the corresponding size increase of the SC to prevent singularity. Without further infalling leptons, the short-range repulsive force from the super force field disintegrates the SLS to generate the SLS plasma fragments (electron-positron pair plasma) detaching from the SC. When the amount of the SLS plasma fragments is high enough, some SLS plasma fragments in the inner part of the SAD generating the relativistic SAD jet through the process similar to the Blandford-Znajek process [16], and some SLS plasma fragments in the SE generate the coincident relativistic SE jet through the process similar to the Penrose Process [17]. The result is the

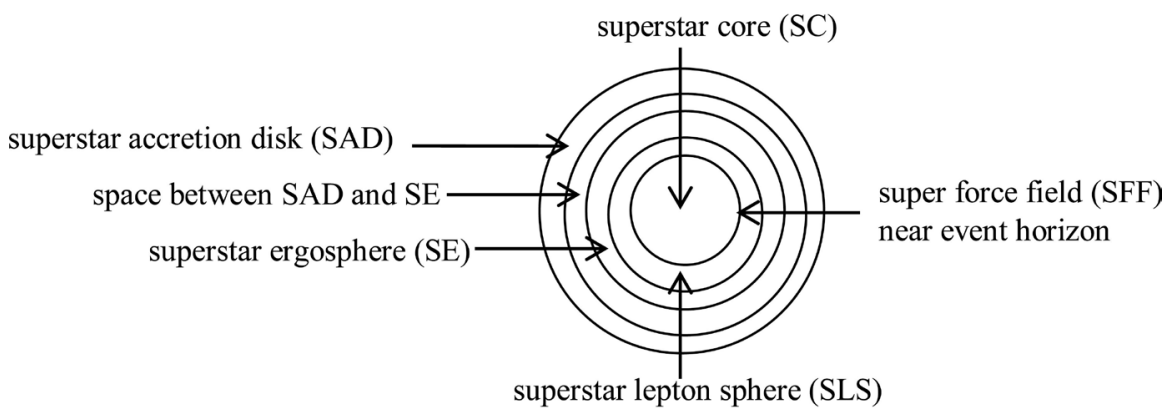

Figure 2. The structure of superstar. 
two-jet model based on supermassive superstar.

In the conventional Blandford-Znajek process for the jet from supermassive black hole, the energy of the relativistic jet is extracted from magnetic fields around an accretion disk, which are dragged and twisted by the spin of the supermassive black hole. The Blandford-Znajek process requires an accretion disk with a strong poloidal magnetic field around a rotating supermassive black hole. In the conventional Penrose Process for the jet from supermassive black hole, the energy of the relativistic jet is extracted from the negative-energy particles produced in the ergosphere. The Penrose Process requires the breakup (spontaneous fission) of particles at relativistic speeds in opposite directions in the ergosphere. Parfrey, Philippov, and Cerutti recently present general-relativistic collisionless plasma simulations [18] of rotating supermassive black hole magnetospheres which begin from vacuum, inject electron-positron pair plasma near the event horizon, and reach steady states with electromagnetically powered Blandford-Znajek jets that occurs at north and south poles. Near the supermassive black hole's midsection in the ergosphere, magnetic field lines operating in opposing directions like a two-lane highway meet at the equator. This congregation causes the lines to twist and tangle. In the space between these bundles, the electric field accelerates particles. Some fly outward, following a curved trajectory into the peripheries of the polar jets. Others speed into the supermassive black hole, resulting in the Penrose process. Overall, the simulations suggest that roughly $80 \%$ of the jet's energy comes from Blandford-Znajekwith the remaining $20 \%$ originating from the Penrose process. The computer simulation by Parfrey, Philippov, and Cerutti produces the two-jet model based on supermassive black hole.

For the two-jet model based on superstar, the main energy sources of both SAD jet and SE jet are the SLS plasma fragments (electron-positron pair plas$\mathrm{ma})$, and the formations of the jets are derived from the rotating SE/SAD. Because the inner portions of the lepton layers on the SE/SAD are rotating more quickly than the outer portions, the magnetic field lines twist violently. This causes the jets of leptons to blast outward at almost the speed of light perpendicularly to the SE/SAD, similar to the Blandford-Znajek process and Penrose process. The SLS plasma fragments provide sufficient energies to produce large scale relativistic jets. Furthermore, the short-range repulsive force of the super force field also avoids the adsorption of the SLS plasma fragments by the SC, unlike the Blandford-Znajek process and Penrose process with supermassive black hole to adsorb plasma. As a result, AGN based on supermassive superstar allows large-scale relativistic jets. Some computer simulations of AGN jet based on supermassive black hole that do not have unlimited plasma and the Penrose process as the simulation by Parfrey, Philippov, and Cerutti cannot generate large-scale jets [19].

The jet from the SLS plasma fragments is similar to the jet from a symbiotic binary which usually contains a white dwarf with a companion red giant [20]. A 
red giant loses its material through the flow to the white dwarf nearby via an accretion disk. Some symbiotic stars have jets which are bi-polar and extend from both poles of the white dwarf. Therefore, the red giant is like the SLS as the source of the SLS plasma fragments, while the accretion disk of the white dwarf is like the SE/SAD. However, the strong gravity of supermassive superstar prevents the escape of material through the jet formation directly and initially from the infalling star as in a symbiotic binary. The SLS plasma fragments inside the structure of supermassive superstar provide the critical mass in a small area to generate a large scale jet.

The distance between the SAD and the SAD jet is much larger than the distance between the SC and the SE jet, so the leptons from the SAD travels longer distance from the SAD than from the SC to form the jets in the high-density BLR. As a result, the SAD jet is the slow jet with the low Lorentz factor, and the SE jet is the fast jet with the high Lorentz factor. Due to the longer distance between the SAD and the SAD jet than the distance between the SC and the SE jet, the radius of the SAD jet is larger than the radius of the SE jet. Therefore, the fast and narrow SE jet is inside the coincident slow and broad SAD jet as the two-jet model in Figure 3.

In this two-jet model, the relativistic leptons in the fast $\mathrm{SE}$ jet up-scatter radiation from the slow moving SAD jet to produce gamma-ray as in the Synchrotron Self Compton (SSC) model [21]. In the SSC model, the seed photons for the Inverse Compton (IC) process producing gamma-ray are the synchrotron photons produced by the relativistic leptons. This two-jet model is similar to the two-blob model proposed by Georganopoulos et al. [22] in which radiation from the slow blob II is up-scattered by the relativistic mono-directional leptons in the fast blob I. The two-blob model by Georganopoulos et al. fits the SED well.

The slow SAD jet generates mostly the low frequency emissions and occasionally low-energy gamma-ray emissions through the inverse Campton scattering with the photons in the high-density BLR, while under the protection by the SAD jet, the fast SE jet inside the slow SAD jet generates the VHE SSC emission without the attenuation by the high-density photons in the BLR of FSRQ. Therefore, the two-jet model of the SAD jet and the SE jet provides the solution for the relativistic producing the VHE gamma-ray emissions in FSRQ.

The two-jet model also explains VHE gamma-rays [23] in FR1 type radio galaxies which jets aligned at large angles to the line of sight. In the two-jet model, the SAD jet producing VHE gamma-rays in FR1 type radio galaxies is the very

$\mathrm{SAD}, \mathrm{SE}, \mathrm{SC}$, and SLS plasma fragments

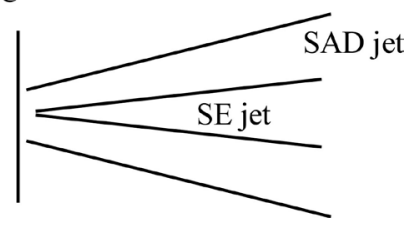

Figure 3. The two-jet model: the fast and narrow SE jet from the SC is inside of the slow and broad SAD jet from the SAD. 
slow SAD jet through the very high-density BLR. As the very slow SAD jet moves through the very high-density BRL, the fast-moving photons in the very high-density BLR deaccelerate the very slow SAD jet, and change the mono-directional leptons in the SAD jet into multi-directional leptons. Subsequently, some multi-directional leptons in the SAD jet in the narrow line region produce VHE gamma-rays by up-scattering mono-directional soft radiation from the SE jet preferentially in the direction opposite to the SE jet motion. Therefore, VHE gamma-rays which are produced in the SAD jet without the attenuation from the photons in the low-density narrow line region can be emitted at a relatively large angle to the jet axis in the observer's reference frame. This two-jet model is similar to the two-blob model proposed by Banasinski and Bednarek [24] in which radiation from the fast blob I is up-scattered by the relativistic isotropic (multi-directional) leptons in the slow blob II. The two-blob model by Banasinski and Bednarek fits the SED well. Therefore, the two-jet model of the very slow SAD jet and the SE jet provides the solution for the relativistic jet producing the VHE gamma-ray emissions in FR1 type radio galaxies.

The currents carried by the AGN jets can be probed using maps of the Faraday rotation measure (RM), since a jet current are accompanied by a toroidal magnetic field, which gives rise to a systematic change in the RM across the jet [25]. The collected data indicate that AGN jets reveal strong signs of the spine-sheath jet structure with a central spine of orthogonal magnetic field and a sheath of longitudinal magnetic field along the edges of the jet [26]. They suggest that the inner region of the jet spine consists of a low-density and fast-moving gas, while the outer region of the jet sheath consists of a denser and slower moving gas [27] The spine-sheath jet structure can also be explained by the coaxial cable proposed by Gabuzda [28] [29]. Like coaxial cable with the inner conductor and the outer shield sharing a geometric axis, an AGN jet has an inward current along its axis and an outward current in a more extended sheath-like region surrounding the jet. The current typically flows inward along the jet axis and outward in a more extended region surrounding the jet, typical to the current structure of a coaxial cable, accompanied by a self-consistent system of nested helical magnetic fields, whose toroidal components give rise to the observed transverse RM gradients. These data conclusively demonstrate the existence of the coaxial currents of AGN jets. The origin of the spine-sheath jet structure can be explained by the two-jet model. The spine (inward current) and the sheath (outward current) in the spine-sheath jet structure model are derived from the SE jet and SAD jet, respectively, in the two-jet model.

The amount of the SLS plasma fragments is determined by the thicknesses of the SLS derived from the mass of infalling star and interstellar material. The SLS plasma fragments stay around the SE/SAD through gravity until they escape through the relativistic jets. The jets have limited capacities to carry leptons. Therefore, the timescale of the jet is determined by the amount of the SLS plasma fragments which is determined by the thickness of the SLS derived from the 
mass of infalling star and interstellar material. When the amount of the SLS plasma fragments becomes insufficient to generate the jet, the jet ends. The SLS plasma fragment remnants (electron-positron pairs) become the fast-moving photons in the BLR. The SAD, the SE, and the SLS maintain the minimum amounts of leptons in the equilibrium state among gravity, angular momentum, and the short-range repulsive force of the super force field. The fast-moving photons from the SLS fragment plasma remnants in the BLR gradually move away from the BLR. This process repeats for another occurrence of infalling star and interstellar material. The amount of the photons from the SLS plasma fragment remnants is determined by the frequency of the occurrence of infalling star and interstellar material. The jetted AGN with frequent occurrences of infalling star and interstellar material are FSRQs with the high amount of photons from the SLS plasma fragment remnants in the BLR, while the jetted AGN with infrequent occurrences are BL Lacertae objects with the low amount of photons from the SLS plasma fragment remnants in the BLR. The result is the blazar sequence as the sequence of the amounts of photons surrounding the jets [30].

\section{Conclusions}

In this paper we present solutions for the problems of singularity and the relativistic jet in AGN (Active Galactic Nuclei) based on supermassive black hole with singularity. The five-zone structure of superstar from inside to outside consists of the Singularity-Free Superstar Core (SC), the short-range repulsive super force field (SFF) near the event horizon, the superstar lepton sphere (SLS) containing infalling leptons (electron-positron pairs), the superstar ergosphere (SE), and the superstar accretion disk (SAD). As in the Meissner effect in superconductor, the short-range SFF repulses leptons in the SLS preventing singularity, while infalling leptons from the SAD and the SE continue to enter the SLS through the strong gravity of the SC. When the density at the bottom of the SLS reaches the critical density, leptons fall into the SC with the corresponding size increase of the SC to prevent singularity.

Without further infalling leptons, the short-range repulsive force from the SFF disintegrates the SLS into the SLS plasma fragments (electron-positron pair plasma), detaching from the SC. Some SLS plasma fragments in the SAD generate the broad relativistic SAD jet, and some SLS plasma fragments in the SE generate the coincident narrow relativistic SE jet. In this two-jet model (the origin of the spine-sheath jet structure), protected by the SAD jet, the fast and narrow SE jet inside the slow and broad SAD jet generates the VHE (very high energy $\geq 100$ $\mathrm{GeV}$ ) Synchrotron Self Compton (SSC) gamma-ray emission without the attenuation by the photons in the BLR (broad line region) of flat spectrum radio quasar (FSRQ). The two-jet model of the very slow SAD jet and the SE jet also provides the solution for the relativistic jet producing the VHE gamma-ray emissions in FR1 type radio galaxies.

An important theory on the jet structure is the spine-sheath jet structure. The 
origin of the spine-sheath jet structure can be explained by the two-jet model. The spine and the sheath in the spine-sheath jet structure model are derived from the SE jet and SAD jet, respectively, in the two-jet model. The timescale of the jet is determined by the amount of the SLS plasma fragments which is determined by the thickness of the SLS derived from the mass of infalling star and interstellar material. When the amount of the SLS plasma fragments becomes insufficient to generate the jet, the jet ends. The SLS plasma fragment remnants (electron-positron pairs) become the fast-moving photons in the BLR. The jetted AGN with frequent occurrences of infalling star and interstellar material are FSRQs with the high amount of photons from the SLS plasma fragment remnants in the BLR, while the jetted AGN with infrequent occurrences are BL Lacertae objects with the low amount of photons from the SLS plasma fragment remnants in the BLR. The result is the blazar sequence as the sequence of the amounts of photons surrounding the jets. In conclusion, AGN based on supermassive superstar provide the solutions for singularity, VHE gamma-ray emissions in FSRQs and FR1 type radio galaxies, AGN jet structure, and AGN jet type.

\section{Conflicts of Interest}

The author declares no conflicts of interest regarding the publication of this paper.

\section{References}

[1] Mazur, P. and Mottola, E. (2004) Gravitational Vacuum Condensate Stars. Proceedings of the National Academy of Science of the United States of America, 101, 9545-9550. https://doi.org/10.1073/pnas.0402717101

[2] Mazur, P. and Mottola, E. (2015) Surface Tension and Negative Pressure Interior of a Non-Singular 'Black Hole'. Classical and Quantum Gravity, 32, Article ID: 215024. https://doi.org/10.1088/0264-9381/32/21/215024

[3] Chung, D. and Krasnoholovets, V. (2013) Singularity-Free Superstar as an Alternative to Black Hole and Gravastar. Journal of Modern Physics, 4, 1-6. https://doi.org/10.4236/jmp.2013.47A1001

[4] Chung, D. (2015) The Basic Cause of Superconductivity. Journal of Modern Physics, 6, 26-36. https://doi.org/10.4236/jmp.2015.61005

[5] Kazanas, D. (2012) Toward a Unified AGN Structure. Astronomical Review, 7, 92-123. https://doi.org/10.1080/21672857.2012.11519707

[6] Urry, C. and Padovani, P. (1995) Unified Schemes for Radio-Loud Active Galactic Nuclei. Publications of the Astronomical Society of the Pacific, 107, 803-845. https://doi.org/10.1086/133630

[7] Sikora, M., Begelman, M. and Rees, M. (1994) Comptonization of Diffuse Ambient Radiation by a Relativistic Jet: The Source of Gamma Rays from Blazars? Astrophysical Journal, 421, 153-162. https://doi.org/10.1086/173633

[8] Sikora, M., Stawarz, Ł., Moderski, R., Nalewajko, K. and Madejski, G.M. (2009) Constraining Emission Models of Luminous Blazar Sources. The Astrophysical Journal, 704, 38-50. https://doi.org/10.1088/0004-637X/704/1/38 
[9] Costamante, L., Cutini, S., Tosti, G., Antolini, E. and Tramacere, A. (2018) On the Origin of Gamma Rays in Fermi Blazars: Beyond the Broad Line Region. Monthly Notices of the Royal Astronomical Society, 477, 4749-4767. https://doi.org/10.1093/mnras/sty887

[10] Capozziello, S., Lambiase, G. and Scarpetta, G. (2000) Generalized Uncertainty Principle from QuantumGeometry. International Journal of Theoritical Physics, 39, 15-22. https://doi.org/10.1023/A:1003634814685

[11] Iyer, B. and Bhawal, B. (1999) Black Holes Gravitational Radiation and the Universe. Springer, New York. https://doi.org/10.1007/978-94-017-0934-7

[12] Chaisson, E. (1990) Relatively Speaking: Relativity, Black Holes, and the Fate of the Universe. W. W. Norton \& Company, New York.

[13] Teo, E. (2003) Spherical Photon Orbits around a Kerr Black Hole. General Relativity and Gravitation, 35, 1909-1926. https://doi.org/10.1023/A:1026286607562

[14] Visser, M. and Williams, T. (2008) The Kerr Spacetime: A Brief Introduction. Oxford Scholarship Online, Oxford. https://doi.org/10.1093/acprof:oso/9780195309386.003.0001

[15] Wagoner, R. (2008) Relativistic and Newtonian Diskoseismology. New Astronomy Reviews, 51, 828-834. https://doi.org/10.1016/j.newar.2008.03.012

[16] Blandford, R. and Znajek, R. (1977) Electromagnetic Extraction of Energy from Kerr Black Holes. Monthly Notices of the Royal Astronomical Society, 179, 433-456. https://doi.org/10.1093/mnras/179.3.433

[17] Penrose, R. (1969) Gravitational Collapse: The Role of General Relativity. La Rivista del Nuovo Cimento, 1, 252-276.

[18] Parfrey, K., Philippov, A. and Cerutti, B. (2019) First-Principles Plasma Simulations of Black-Hole Jet Launching. Physics Review Letters, 122, Article ID: 035101. https://doi.org/10.1103/PhysRevLett.122.035101

[19] Komissarov, S. (2005) Observations of the Blandford-Znajek Process and the Magnetohydrodynamic Penrose Process in Computer Simulations of Black Hole Magnetospheres. Monthly Notices of the Royal Astronomical Society, 359, 801-808. https://doi.org/10.1111/j.1365-2966.2005.08974.x

[20] Mikołajewska, J. (2002) Orbital and Stellar Parameters of Symbiotic Stars. ASP Conference Series, 303, 9-24

[21] Jones, T., O’dell, S. and Stein, W. (1974) Physics of Compact Nonthermal Sources. I. Theory of Radiation Processes. Astrophysical Journal, 188, 353-368. https://doi.org/10.1086/152724

[22] Georganopoulos, M., Perlman, E.S. and Kazanas, D. (2005) Is the Core of M87 the Source of Its TeV Emission? Implications for Unified Schemes. The Astrophysical Journal, 634, L33-L36. https://doi.org/10.1086/498714

[23] Aliu, E., et al. (2012) Veritas Observations of Day-Scale Flaring of M 87 in 2010 April. The Astrophysical Journal, 746, 141.

[24] Banasinski, P. and Bednarek, W. (2018) Two Blobs in a Jet Model for the $\gamma$-Ray Emission in Radio Galaxies. The Astrophysical Journal, 864, 128. https://doi.org/10.3847/1538-4357/aad4fa

[25] Broderick, A. and Loeb, A. (2009) Signatures of Relativistic Helical Motion in the Rotation Measures of Active Galactic Nucleus Jets. The Astrophysical Journal Letters, 703, L104-L108. https://doi.org/10.1088/0004-637X/703/2/L104

[26] Gabuzda. D., Reichstein, A. and O’Neill, E. (2014) Are Spine-Sheath Polarization Structures in the Jets of Active Galactic Nuclei Associated with Helical Magnetic 
Fields? Monthly Notices of the Royal Astronomical Society, 444, 172-184. https://doi.org/10.1093/mnras/stu1381

[27] Walg, S., et al. (2013) Relativistic AGN Jets I. The Delicate Interplay between Jet Structure, Cocoon Morphology and Jet-Head Propagation. Monthly Notices of the Royal Astronomical Society, 433, 1453-1478. https://doi.org/10.1093/mnras/stt823

[28] Gabuzda, D., Nagle, M. and Roche, N. (2018) The Jets of AGN as Giant Coaxial Cables. Astronomy \& Astrophysics, 612, Article No. A67. https://doi.org/10.1051/0004-6361/201732136

[29] Gabuzda, D. (2019) Evidence for Helical Magnetic Fields Associated with AGN Jets and the Action of a Cosmic Battery. Galaxies, 7, 14 p. https://doi.org/10.3390/galaxies7010005

[30] Fossati, G., et al. (1998) A Unifying View of the Spectral Energy Distributions of Blazars. Monthly Notices of the Royal Astronomical Society, 299, 433-448. https://doi.org/10.1046/j.1365-8711.1998.01828.x 